LBNL-52910

To be published in IEEE Trans. Plasma Sci.

\title{
Measurement of Secondary Electron Emission Yields*
}

\author{
Y. Chutopa ${ }^{\mathrm{a}}$, B. Yotsombat ${ }^{\mathrm{a}}$ and I. G. Brown ${ }^{\mathrm{b}}$ \\ ${ }^{a}$ Department of Physics, Chiang Mai University, Chiang Mai 50200, Thailand \\ ${ }^{b}$ Lawrence Berkeley National Laboratory, Berkeley, California 94720, USA
}

June 2003

* Supported in part by the U.S. Department of Energy under contract number DE-AC0376SF00098. 


\title{
Measurement of Secondary Electron Emission Yields
}

\author{
Y. Chutopa ${ }^{\mathrm{a}}$, B. Yotsombat ${ }^{\mathrm{a}}$, and I. G. Brown ${ }^{\mathrm{b}}$ \\ ${ }^{a}$ Department of Physics, Chiang Mai University, Chiang Mai 50200, Thailand \\ ${ }^{b}$ Lawrence Berkeley National Laboratory, Berkeley, California 94720, USA
}

\section{Abstract}

We describe a method for the measurement of secondary electron emission coefficients, and demonstrate the use of this approach for the measurement of secondary electron yields for titanium, copper and carbon ions incident upon an aluminum target. The method is timeresolved in that a series of measurements can be obtained within a single ion beam pulse of several hundred microseconds duration. The metal ion beams were produced with a vacuum arc ion source, and the ratio of secondary electron current to incident ion current was determined using a Faraday cup with fast control of the electron suppressor voltage. The method is relatively simple and readily applied, and is suitable for measurements over a wide parameter range. The secondary yields obtained in the present work are of relevance to the measurement of ion current and implantation dose in plasma immersion ion implantation.

\footnotetext{
* Corresponding author. E-mail address: banchoby@hotmail.com
} 


\section{INTRODUCTION}

The measurement of secondary electron emission coefficient for ions incident on various metallic targets as a function of ion energy is important for the understanding of ion-solid interactions in many different areas. The conventional approach to the measurement of secondary electron yields makes use of polished and ultra-clean metal target surfaces in ultrahigh vacuum systems using mass-analyzed ion beam techniques [1-8]. Alonso and coworkers [2] measured the charge-state dependence of ion-induced electron emission from aluminum using this approach. Holmen et al. [3] determined the electron yields for ions incident on polycrystalline copper over a wide energy range, and Svensson et al. [4] measured yields from incident protons and nobles gas ions incident upon aluminum targets. Typically, for incident ion energies in the $10-50 \mathrm{keV}$ range and for a wide range of metallic target materials, the secondary electron emission coefficient, $\gamma$, lies in the broad range 1 to 10 electrons/ion.

Much plasma science and technology makes use of surfaces that are neither polished nor ultra-clean and systems that are not at ultra-high vacuum, and thus the secondary electron yields obtained using the standard methods may not realistically apply to plasma-based processes with strong ion-surface interactions. Hence these results may be less valuable for understanding plasma-based processes as used in the plasma laboratory and in industry. The growing application of plasma immersion ion implantation (PIII or $\mathrm{PI}^{3}$ ) [8-11] as a surface modification tool in research and industry provides a need for specific secondary electron yield data. In PIII an electrically conducting target is immersed in a plasma and repetitively pulsebiased to high negative voltage so as to accelerate ions from the plasma across the high voltage sheath that is formed surrounding the target and into the implantation target. The ion implantation dose is estimated from the integrated target current. However, this measured current is the sum of the positive ion current incident upon the target and the secondary electron current emitted from the target, and it is common that the secondary electron current 
can be several times greater than the ion current. Thus, in order to correctly estimate the ion dose, the secondary electron yield must be known with some precision.

Several groups have used more realistic techniques to measure secondary electron emission coefficients. Szapiro et al. [12-13] measured secondary electron yields for He, Ar and $\mathrm{Ne}$ ions on targets conditioned in a glow discharge plasma by switching between glow discharge and high vacuum environments. The values of $\gamma$ obtained by this method were about 2 - 3 times greater than those obtained using the standard methods (ultra-clean surfaces in ultra-high vacuum with mass-analyzed ion beams) and depended strongly on the discharge conditions. En and Cheung [14] made use of a PIII technique. Shamim et al. [15] used a Rogowski transformer to measure the current drawn by a target located in a plasma immersion environment to determine the secondary electron yields for a number of ion-target combinations for ion energies from 20 to $40 \mathrm{keV}$. The values of $\gamma$ measured by En and Cheung were comparable with those reported by Szapiro et al., while Shamim and coworkers found values of $\gamma$ that were about twice those of Szapiro. Qin et al. [16] found that these indirect methods ignore the phenomenon of plasma density enhancement during the high voltage pulse, which in turn increases the secondary yield, and developed a direct method based on a Faraday dosimetry technique for measuring the incident ion flux that automatically incorporates the effect of plasma density enhancement. Qin et al. obtained secondary yields with values lower than those obtained by previous PIII methods but still higher than the standard methods.

It is not uncommon for multiply-charged ions to be formed in some kinds of plasma discharges, most especially in metal plasmas formed by vacuum arc discharges. Vacuum arc metal plasmas have been used for plasma immersion ion implantation by a number of workers [10,11,17-19]. In this case the quantification of secondary electron yield is complicated both by the effects of multiply stripped ions as well as by the need for data for the case when the incident ions are metal ions. In the work described here, we have used a vacuum arc ion source 
$[20,21]$ to form energetic beams of multiply charged metal ions. Using this kind of ion source together with a specially-designed Faraday cup in which the secondary electron suppression voltage is pulsed to various levels during the ion beam pulse, we have developed a timeresolved approach that allows us to obtain the secondary electron yields as a function of time throughout the ion beam pulse. The metal ion beam produced by a vacuum arc ion source has the same ion composition as is the metal plasma used for metal plasma immersion ion implantation, and thus the secondary electron yields measured in this way are directly applicable for interpreting the measured target current, and thus estimating the ion implantation dose, in metal plasma immersion ion implantation processes.

\section{APPROACH}

The secondary electron coefficient $\gamma$ is defined as the number of electrons ejected from the surface per incident bombarding ion. For the case of singly charged ions $\gamma$ can be expressed simply as

$$
\gamma=\frac{I_{e}}{I_{i}}
$$

where $I_{e}$ is the secondary electron current and $I_{i}$ is the incident ion current. For the case of multiply charged ions, however, we must distinguish between electrical current $I_{(e l e c)}$ and particle current $I_{(\text {part })}$. For multiply charged ions of charge state $q, I_{(\text {part })}=I_{(\text {elec })} / q$, and $\gamma$ is then given by

$$
\gamma=\frac{q I_{e}}{I_{i}} .
$$

Consider a Faraday cup with electrostatic suppression of secondary electrons, upon which an energetic beam of ions is incident. For the case of complete suppression of secondary electrons (all secondaries returned to the collector) the measured cup current is equal to the 
incident ion current $I_{i}$, while for the case of no secondary electron suppression (all secondaries removed from the collector) the total measured cup current $I_{T}$ is equal to the sum of the ion current and the secondary electron current, $I_{T}=I_{i}+I_{e}$. From Eq. (2) $I_{e}=\gamma I_{i} / q$, and we obtain

$$
\gamma=q\left(\frac{I_{T}}{I_{i}}-1\right) .
$$

Thus we can obtain the secondary electron coefficient $\gamma$ by measuring the Faraday cup currents for given constant incident ion flux, for the cases when there is zero suppression of secondaries (no bias applied to the electron suppressor) and when there is complete suppression of secondaries (adequate bias applied to the electron suppressor for full electron suppression). For the mean charge state $\bar{q}$ for the ions produced by a metal vapor vacuum arc ion source, as used here, have been measured and are well know [20,22]. The suppressor bias voltage necessary for complete suppression of secondary electrons in the Faraday cup is determined experimentally by varying the suppressor bias while monitoring the cup current. As the magnitude of the (negative) suppressor voltage is increased, the cup current decreases until a saturation is reached; this is the point at which all secondary electrons are suppressed (reflected and returned to the collector).

The Faraday cup operational technique used here is different from the usual. Conventionally, electron suppression within the Faraday cup is used so as to obtain the true ion current. Here we switch the suppressor bias voltage rapidly between two specific levels so as to obtain measurements of $I_{i}$ and of $I_{i}+I_{e}$, both from the Faraday cup signal. 


\section{EXPERIMENTAL METHOD}

The work was carried out using an ion beam facility with a specially designed Faraday cup that allowed fast switching of the suppressor bias voltage. The ion species produced could be changed, and the secondary electron target was mounted inside the Faraday cup. The vacuum system pressure was about $9 \times 10^{-6}$ Torr.

\section{A. Ion Beam}

A vacuum arc ion source (Mevva), made in our laboratory and described in detail elsewhere [21], was used to produce energetic beams of carbon, titanium and copper ions. The source was operated in pulsed mode, with pulse width about $400 \mu$ s. The ion species produced (C, $\mathrm{Ti}$ or $\mathrm{Cu}$ ) was changed by changing the arc cathode material; arc current was $130 \mathrm{~A}$. Ion extraction voltage could be controlled up to $24 \mathrm{kV}$. It is known that ions produced in a vacuum arc ion source are in general multiply ionized [20,22]. Titanium, for example, has a spectrum that is $10 \% \mathrm{Ti}^{+}, 75 \% \mathrm{Ti}^{2+}$ and $15 \% \mathrm{Ti}^{3+}$ and that is unchanged over a wide range of ion source operational parameters. The ion energy is given by $E_{i}=q V_{\text {ext }}$, where $q$ is the ion charge state and $V_{\text {ext }}$ the ion source extraction voltage. It is convenient, and there is little loss of precision for many applications, if we define the mean ion beam energy as $\bar{E}=\bar{q} V_{\text {ext }}$. Mean ion charge states for vacuum arc plasmas of almost all of the metals of the Periodic Table have been measured and tabulated $[20,22]$. For $\mathrm{C}, \mathrm{Ti}$ and $\mathrm{Cu}$ the mean ion charge states are 1.0, 2.1 and 2.0 , respectively.

\section{B. Faraday Cup}

The Faraday cup was fabricated from aluminum. A schematic outline of the Faraday cup is shown in Fig. 1(a), and photographs of the conical suppressor and of the entire assembly in Figs. 1(b) and (c), respectively. An additional planar suppressor plate is located in front of the conical suppressor, in accordance with McKenna's design [23]. This additional suppressor was 
DC biased as shown in Fig 2; we found this necessary for correct operation of the Faraday cup. The conical suppressor has three rings with triangular cross-section machined into the inside conical metal surface. This compares with the more usual kind of Faraday cup that employs a simple hollow cylinder suppressor, as for example used by Jamba [19]. The beam defining entrance aperture serves to prevent the incoming ions from directly impacting the suppressor, and has a diameter of $5.2 \mathrm{~mm}$. The ion collection of our Faraday cup was compared with another Faraday cup with length to diameter ratio 70.3 , known to trap $>99 \%$ of incident ions, and the agreement between the two systems was within $8 \%$. The suppressor bias voltage required for complete secondary electron suppression was determined to be less than $-300 \mathrm{~V}$; we thus used $-300 \mathrm{~V}$ as bias to obtain our $I_{i}$ value. The $I_{i}+I_{e}$ signal was obtained as the collector current when the suppressor bias was $0 \mathrm{~V}$.

\section{Electronics System}

We found in preliminary work that there is a high level of electromagnetic noise generated by the Mevva ion source system, and that pick-up of these unwanted signals can perturb and possibly damage other parts of the overall electronics. We therefore developed an optical signal coupling system that served the dual purposes of removing much of the unwanted high level of pick-up signal and also allowed us to incorporate a novel kind of time resolved approach into our measurement of secondary electron coefficient. An optical signal coupling system and high voltage transistor switching circuit for the Faraday cup suppressor are employed as shown in Fig. 2. A laser pointer driven by a 2N2219 NPN transistor was initially used for the optical coupling but we found it not suitable for repetitive operation, and we turned to using a system incorporating a super-bright LED, photo diodes, and focusing lenses. The LED generates a signal that coincides with the firing of the pulsed ion beam, thus providing the appropriate timing for applying the pulsed bias to the Faraday cup suppressor. However, by inserting additional electronics (the "Intermediate unit" shown in Fig. 2) between the LED pulse and the pulse generator for the Faraday cup bias, we were able to generate multiple bias 
pulses within the one ion beam pulse. In this way a time-resolved feature of the measurement is introduced. We found it convenient to use a standard PC computer for this purpose, rather than a specially made electronics circuit. The PC provides a highly flexible means of generating the required multiple pulse signals with their appropriate delays; we used a PC with a $1 \mathrm{GHz}$ CPU operating with Microsoft QuickBasic for signal control. For an ion beam pulse width of $400 \mu \mathrm{s}$ we chose to generate and use three suppressor bias pulses. These pulses were further focused onto a second photodiode and then digitized for driving the 3055T NPN transistor switch. The rise time and fall times of the output square-wave signals induced in the secondary transformer coil were improved (decreased down to $0.2 \mu \mathrm{sec}$ and $0.35 \mu \mathrm{sec}$, respectively) by six serial special number diodes (Type 3939). These square-wave signals drive the HV transistor D2445 that supplies the negative bias voltage pulses to the Faraday cup suppressor. A typical oscillogram of the ion beam Faraday cup signal, with three consecutive bias pulses applied, is shown in Fig. 3.

\section{Measurement Procedure}

The measurements were carried out using singly-charged carbon ions and multiplycharged $\mathrm{Ti}$ and $\mathrm{Cu}$ ions [20,22] produced by a Mevva ion source [20,21] with $400 \mu$ sec pulse duration. Residual contamination due to oil vapor and others impurities coated on the ion source cathode surface were removed by firing the arc several hundred times without ion extraction. The aluminum target (i.e., the ion collector electrode within the Faraday cup) was ultrasonically cleaned in de-ionized water followed by acetone cleaning and baking in vacuum at $\sim 150{ }^{\circ} \mathrm{C}$ for $10 \mathrm{~min}$. The ion beam was repetitively pulsed and the Faraday cup signal monitored, such as typified by Figure 3 for the case of carbon ions. The secondary emission coefficient $\gamma$ was derived from the oscillograms using Eq. (3), where $I_{T}$ is the measured cup current for no applied bias pulse (the higher current value) and $I_{i}$ is the cup current when the bias pulse is applied (the lower current value). Several measurements could be obtained as a 
function of time throughout the pulse. The ion source extraction voltage was varied so as to obtain $\gamma$ as a function of ion energy.

\section{RESULTS AND DisCUSSION}

The variation of mean ion charge state with time for pulsed vacuum arc plasmas has been studied and it is known that for arc parameters quite similar to those of the present work the mean charge state $\bar{q}(t)$ decreases significantly in the first few tens of microseconds, reaching an asymptotic value after about $100-200 \mu \mathrm{s}$. We thus take $\gamma(\mathrm{t}=250 \mu \mathrm{s})$ as a measure of the secondary electron coefficient for given ion beam parameters. These data are summarized in Figure 4, where we plot the secondary yield as a function of mean ion energy for $\mathrm{C}$, $\mathrm{Ti}$ and $\mathrm{Cu}$ ions incident on Al. For carbon ions (for which the mean charge state is always 1.0 here), $\gamma$ varies from 1.0 for $E_{i}=9 \mathrm{keV}$ up to 2.0 for $E_{i}=24 \mathrm{keV}$. For Ti (mean charge state 2.1) $\bar{\gamma}$ ranges from 1.6 at $\bar{E}_{i}=19 \mathrm{keV}$ up to 6.5 for $\bar{E}_{i}=50 \mathrm{keV}$. For $\mathrm{Cu}$ (with mean charge state $\bar{q}=2.0), \bar{\gamma}$ ranges from 2.0 at $\bar{E}_{i}=18 \mathrm{keV}$ up to 7.5 for $\bar{E}_{i}=48 \mathrm{keV}$. We use the symbol $\bar{\gamma}$ to emphasize that our values are an average for the vacuum arc ion charge state distribution; (C: $100 \% 1+$ and $\bar{q}=1 . \mathrm{Ti}: 10 \% 1+, 75 \% 2+, 15 \% 3+$, and $\bar{q}=2.1 . \mathrm{Cu}: 16 \% 1+, 64 \% 2+$, $20 \% 3+$, and $\bar{q}=2.0$ ). We estimate the overall uncertainty of our yield measurements to be about $20-30 \%$.

These values are in reasonable agreement with the very modest amount of comparable data available from the literature. The overwhelming amount of data available on secondary electron emission yields is for the case of gaseous ions, rather than for metal ions as in the present work; this is a consequence of the fact that gas ion beams are in general more easily produced than metal ions beams. We can however make some comparisons. For the case of $\mathrm{C}^{+}$ions on $\mathrm{Al}$, Alonso [2] obtains electron yields $\gamma$ from about 0.5 to 1.6 , compared to our span 
of from 1.0 to 2.0. Alonso's measurements were for the case of an atomically clean Al target whereas our measurements are for a "cleaned" but by no means atomically clean surface; certainly we expect our Al target to have a native oxide layer of thickness at least several tens of angstroms. The data thus imply that the electron yield for an impure Al surface is greater than the yield for a pure Al surface by a factor of about $1.5-2$. We can find no direct comparison from the literature for our $\mathrm{Ti}$ and $\mathrm{Cu}$ results, and can only say that the electron yields, in the approximate range 2 to $8 \mathrm{el} / \mathrm{ion}$, are of the same order as the yields for a range of other incident ions on $\mathrm{Al}$.

\section{CONCLUSION}

We have used a relatively simple ion beam facility, together with a Faraday cup incorporating fast switching of its electron suppressor, to measure secondary electron emission yield as a function of ion energy for $\mathrm{C}, \mathrm{Ti}$ and $\mathrm{Cu}$ ions. The results obtained are in reasonable agreement with those of other workers, and extend the parameter range previously reported in the literature. The measurement technique could be of value for obtaining secondary electron yields for ion/target combinations of relevance to the interpretation of applied dose in plasma immersion ion implantation processes.

\section{Acknowledgements}

We are grateful to the Department of Technical and Economic Cooperation of the Prime Minister's Office of Thailand for financial support for this work. We thank all the Masters Degree students in the Solid State Electronics Laboratory for their assistance, particularly P. Pooljaruansin and P. Toomtho. 


\section{REFERENCES}

[1] G. L. Cano, "Secondary electron emission from $\mathrm{Au}, \mathrm{Mo}$, and $\mathrm{CuBe}$ by high-chargenumber laser-produced metal ions", J. Appl. Phys., vol. 44, no. 12, pp. 5293-5300, Dec. 1973.

[2] E. V. Alonso, R. A. Baragiola, J. Ferron, M. M. Jakas, and A. Oliva-Florio, "Z dependence of ion-induced electron emission from aluminum", Phys. Rev. B, vol. 22, no. 1, pp. 80-87, July 1980.

[3] G. Holmen, B. Svensson, A. Buren, "Ion induced electron emission from polycrystalline copper", Nucl. Instr. Meth., vol. 185, nos. 1-3, pp. 523-532, June 1981.

[4] B. Svensson, G. Holmen, "Electron emission from ion-bombarded aluminum", J. Appl. Phys., vol. 52, no. 11, pp. 6928-6933, Nov. 1981.

[5] M. Vana, H. Kurz, H. P. Winter, F. Aumayr, "Potential and kinetic electron emission from clean gold induced by multicharged nitrogen ions", Nucl. Instr. Meth. Phys. Res. B, vol. 100, nos. 2-3, pp. 402-406, June 1995.

[6] C. Lemell, J. Stockl, J. Burgdorfer, G. Betz, H. P. Winter, and F. Aumayr, "Multicharged ion impact on clean gold(111): suppression of kinetic electron emission in glancing angle scattering", Phys. Rev. Lett. vol. 81, no. 9, pp. 1965-1968, Aug. 1998.

[7] J. Krasa, L. Laska, and M. P. Stockli, D. Fry, "Secondary-electron yield from Au induced by highly charged Ta ions" Nucl. Instr. Meth. Phys. Res. B, vol. 173, no. 3, pp. 281-286, 2001.

[8] J. R. Conrad, J. L. Radtke, R. A. Dodd, and F. J. Worzala, "Plasma source ion-implantation technique for surface modification of materials", J. Appl. Phys., vol. 62, no. 11, pp. 4591-4596, Dec. 1987.

[9] J. V. Mantese, I. G. Brown, N. W. Cheung, and G. A. Collins, "Plasma immersion ion implantation", in: Plasma Processing of Advanced Materials, MRS Bulletin, vol. 21, no. 8, pp. 52-56, 1996. 
[10] See the Proceedings of the International Workshops on Plasma-Based Ion Implantation: J. Vac. Sci. Tech. B, vol. 12, pp. 815-998, 1994; Surf. Coat. Technol., vol. 85, pp. 1-124, 1996; Surf. Coat. Technol., vol. 93, pp. 158-350, 1997; J. Vac. Sci. Tech. vol. 17, pp. 798-899, 1999.

[11] A. Anders (Ed.), Handbook of Plasma Immersion Ion Implantation and Deposition, Wiley, New York, 2000.

[12] B. Szapiro, J. J. Rocca, and T. Prabhuram, "Electron yield of glow discharge cathode materials under helium ion bombardment", Appl. Phys. Lett., vol. 53, no. 5, pp. 358-360, Aug. 1988.

[13] B. Szapiro and J. J. Rocca, "Electron emission from glow-discharge cathode materials due to neon and argon ion bombardment", J. Appl. Phys., vol. 65, no. 9, pp. 3713-3716, May 1989.

[14] W. En and N. W. Cheung, "A new method for determining the secondary electron yield dependence on ion energy for plasma exposed surfaces", IEEE. Trans. Plasma Sci., vol. 24, no. 3, pp. 1184-1187, June 1996.

[15] M. M. Shamim, J. T. Scheuer, R. P. Fetherston, and J. R. Conrad, "Measurement of electron emission due to energetic ion bombardment in plasma source ion implantation", J. Appl. Phys., vol. 70, no. 9, pp. 4756-4759, Nov. 1991.

[16] S. Qin, M. P. Bradley, P. L. Kellerman, and K. Saadatmand, "Measurements of secondary electron emission and plasma density enhancement for plasma exposed surfaces using an optically isolated Faraday cup", Rev. Sci. Instrum. vol. 73, no. 3, pp. 1153-1156, Mar 2002.

[17] I. G. Brown, X. Godechot and K. M. Yu, "Novel metal ion surface modification technique", Appl. Phys. Lett. vol. 58, no. 13, pp. 1392-1394, Apr. 1991.

[18] A. Anders, S. Anders, I. G. Brown, M. R. Dickinson, R. A. MacGill, "Metal plasma immersion ion implantation using vacuum arc plasma sources", J. Vac. Sci. Tech. B, vol. 12, no. 2, pp. 815-820, Mar/Apr 1994. 
[19] I. G. Brown, A. Anders, S. Anders, M. R. Dickinson, I. C. Ivanov, R A. MacGill, X. Yao, and K. M. Yu, "Plasma synthesis of metallic and composite thin films with atomically mixed substrate bonding", Nucl. Instr. Meth. Phys. Res. B, vol. 80/81, part 2, pp. 1281-1287, 1993.

[20] I. G. Brown, "Vacuum arc ion sources", Rev. Sci. Instrum. vol. 65, no. 10, pp. 3061-3081, Oct. 1994.

[21] S. Davydov, B.Yotsombat, L. D. Yu, P. Pramukkul, R. Charoennukul, and T. Vilaithong, "Development of metal vapor vacuum arc ion source in Thailand", Surf. Coat. Technol., vol. 131, nos. 1-3, pp. 39-43, Sep. 2000.

[22] I. G. Brown and X. Godechot, "Vacuum arc ion charge state distributions", IEEE. Trans. Plasma Sci., vol. 19, no. 5, pp. 713-717, Oct. 1991.

[23] C. M. McKenna, "High current dosimetry techniques", Radiation Effects, vol. 44, nos. 1-4, pp. 93-110, 1979. 


\section{Figure Captions}

Fig. 1 (a) Simplified schematic of the Faraday cup.

(b) Conical Faraday cup suppressor.

(c) Rear view of the Faraday cup assembly.

Fig. 2 Simplified schematic of the overall electrical system used.

Fig. 3 Typical oscillogram of the Faraday cup current signal, showing three separate suppressor bias pulses applied during the beam pulse.

Carbon ions on aluminum target. Current scale, $200 \mathrm{mV} / \mathrm{div}$. Sweep speed $100 \mu \mathrm{s} / \mathrm{div}$.

Fig. 4 Secondary electron yield $\gamma$ for $\mathrm{C}$, Ti and $\mathrm{Cu}$ ions on an $\mathrm{Al}$ target as a function of mean ion energy. $\mathrm{C}$, solid triangles $\mathbf{\Lambda}$; Ti, empty circles $\mathrm{\circ}$; $\mathrm{Cu}$, solid circles $\bullet$ 


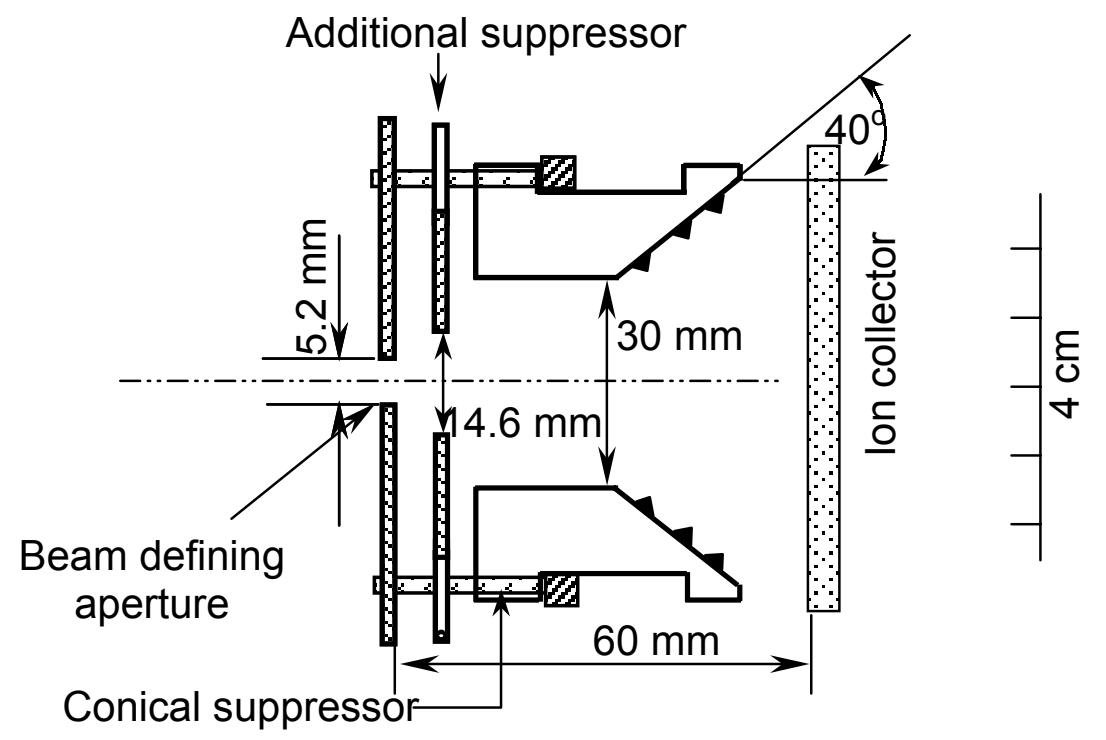

Figure 1(a) 


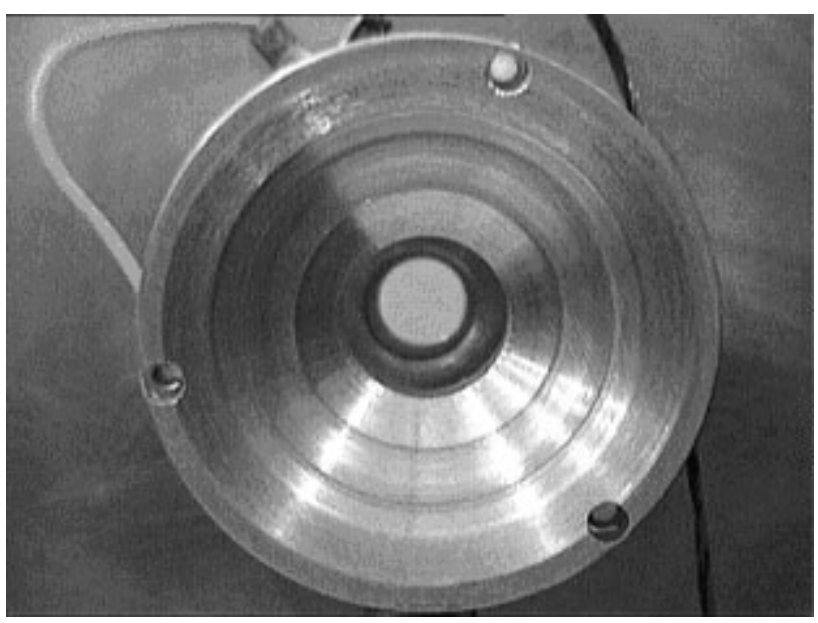

Figure 1(b) 


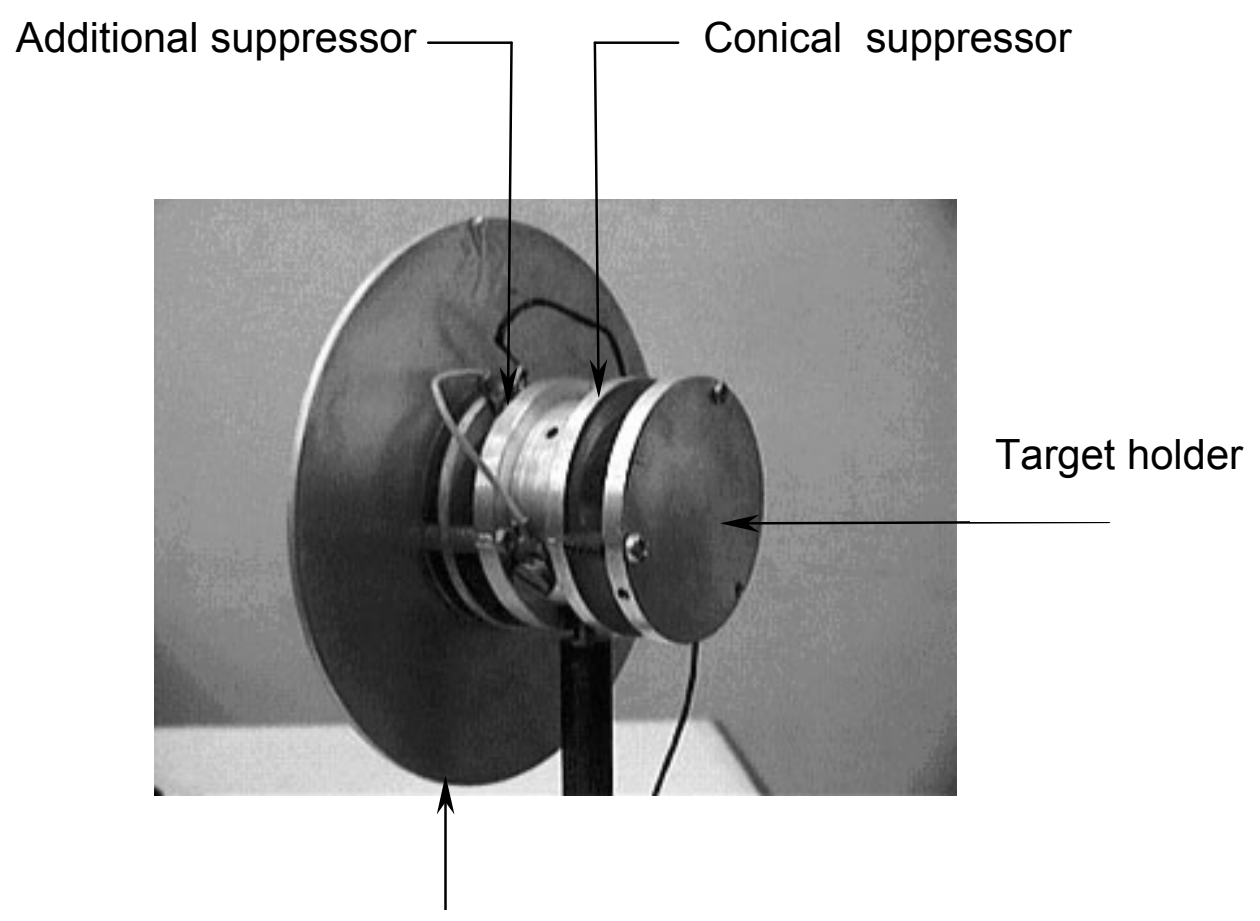

Beam defining aperture plate

Figure 1(c) 


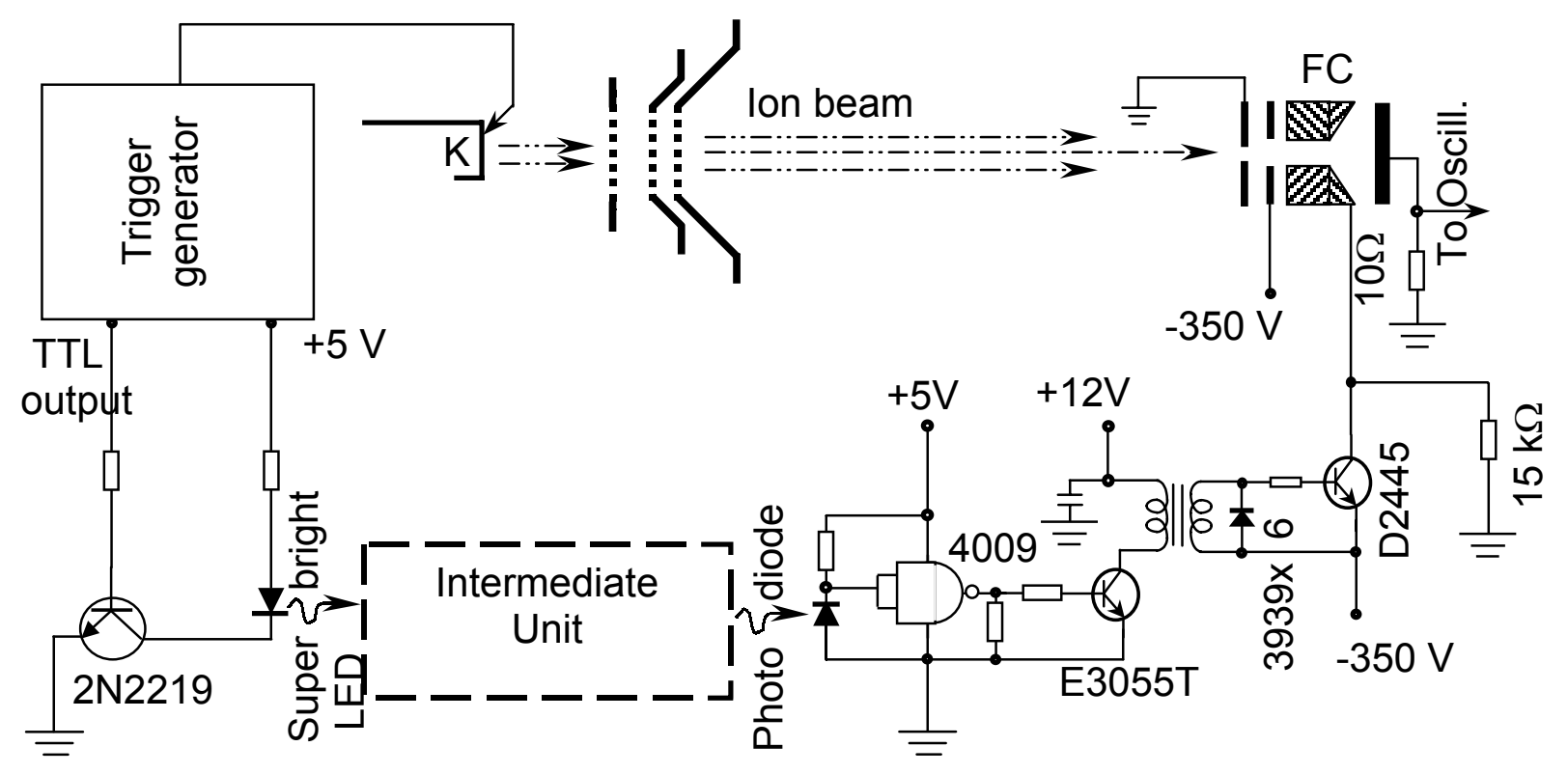

Figure 2 


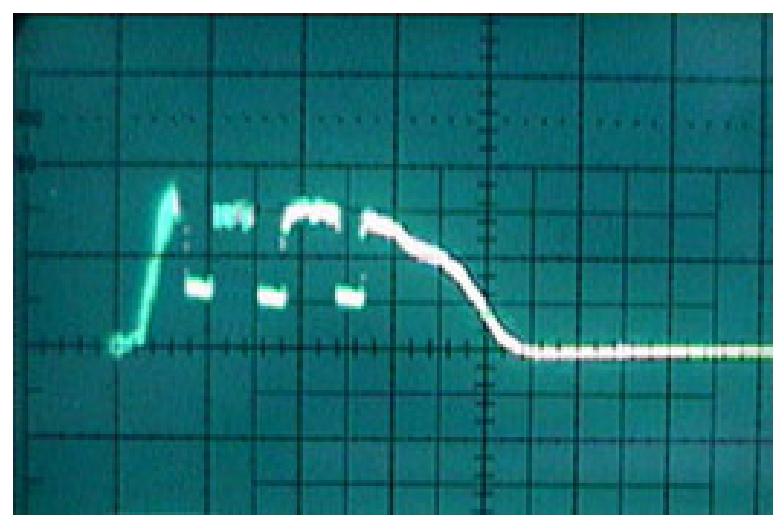

Figure 3 


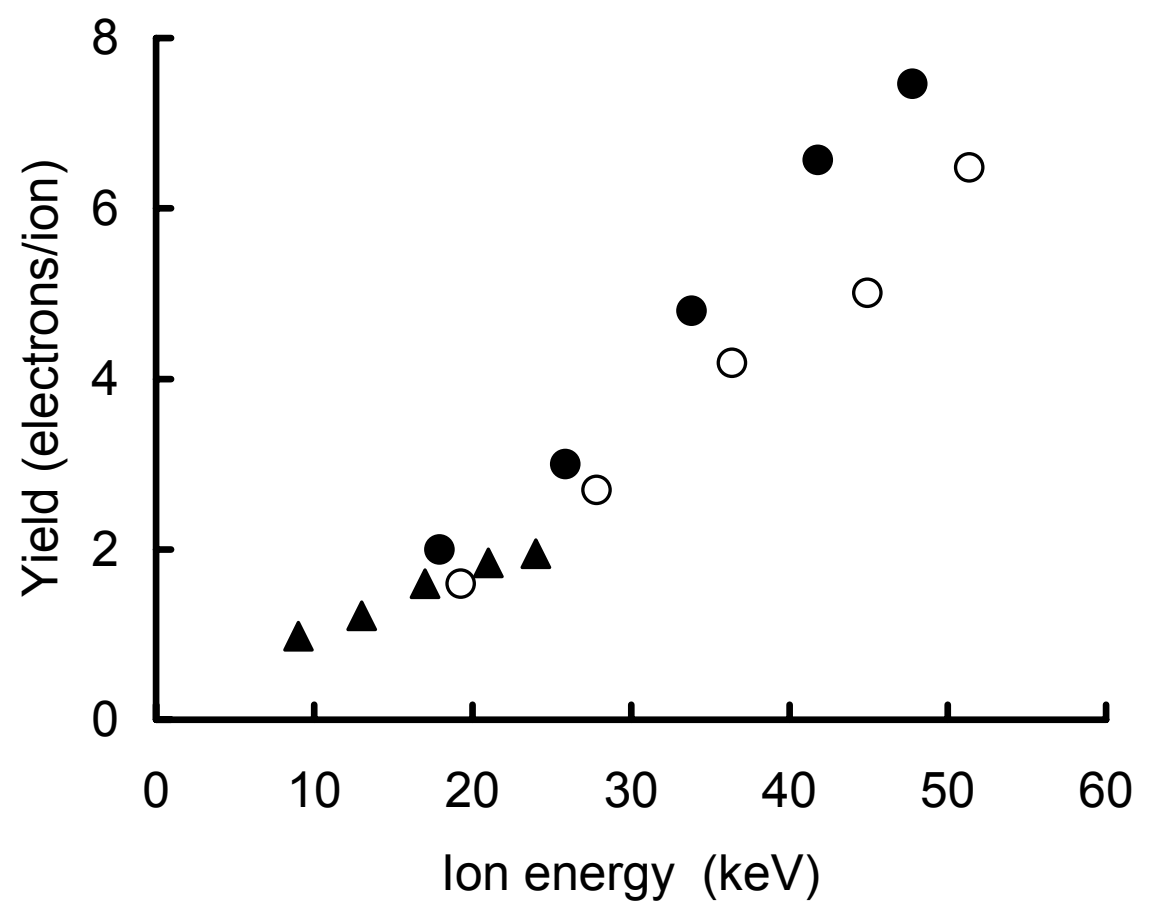

Figure 4 